\title{
Zeros and value sharing results for q-shifts difference and differential polynomials
}

\author{
RAJ SHREE DHAR \\ Higher Education Department, J and K Government \\ Jammu and Kashmir,INDIA \\ dhar.rajshree@jk.gov.in
}

October 15, 2018

\begin{abstract}
In this paper, we consider the zero distributions of q-shift monomials and difference polynomials of meromorphic functions with zero order, that extends the classical Hayman results on the zeros of differential polynomials to q-shift difference polynomials. We also investigate problem of q-shift difference polynomials that share a common value.
\end{abstract}

Mathematics Subject Classification:

2010 : Primary 30D35. Secondary 30D30, 39A05.

Keywords : Entire and Meromorphic function; q-shift; q-difference polynomial; shared values; Nevanlinna theory.

\section{INTRODUCTION:}

A meromorphic (respectively entire) function always means a non-constant function meromorphic (respectively analytic) in the complex plane. Nevanlinna theory of value distribution is concerned with the density of points where a meromorphic function takes a certain value in the complex plane. It is also assumed that the reader is familiar with the basic concepts of Nevanlinna Theory, see e.g. ([1],[2]), such as the characteristic function $T(r, f)$, proximity function $\mathrm{m}(\mathrm{r}, \mathrm{f})$, counting function $\mathrm{N}(\mathrm{r}, \mathrm{f})$ and so on. In addition, $\mathrm{S}(\mathrm{r}, \mathrm{f})$ denotes any quantity that satisfies the condition that $S(r, f)=o(T(r, f))$ as $r$ tends to infinity outside of a possible exceptional set of finite logarithimic measure. In the sequel, a meromorphic function a(z)is called a small function with respect to $\mathrm{f}$ if and only if $\mathrm{T}[\mathrm{r}, \mathrm{a}(\mathrm{z})]=\mathrm{o}(\mathrm{T}(\mathrm{r}, \mathrm{f}))$ as $\mathrm{r}$ tends to infinity outside of a possible exceptional set of finite logarithimic measure. We denote by $\mathrm{S}(\mathrm{f})$, the family of all such small meromorphic functions. 
We say that two meromorphic functions $\mathrm{f}$ and $\mathrm{g}$ share the value a (belonging to extended complex plane) CM (IM)

provided that

$$
f(z) \equiv a
$$

if and only if

$$
g(z) \equiv a,
$$

counting multiplicity (ignoring multiplicity).

\section{DEFINITION 1 :}

Let $\mathrm{c}$ be a non-zero complex costant then for a meromorphic function $\mathrm{f}(\mathrm{z})$, we define its shift by $\mathrm{f}(\mathrm{z}+\mathrm{c})$ and its difference operator by

$$
\Delta_{c} f(z)=f(z+c)-f(z),
$$

In this paper, the q-shift of $\mathrm{f}(\mathrm{z})$ is defined as $\mathrm{f}(\mathrm{qz})$, where $\mathrm{q}$ is non-zero complex constant. The $q$-difference of $f(z)$ is defined as $f(q z)-f(z), q \neq 0,1$. If an equation includes q-shifts or q-differences of $f(z)$, then the equation is called the q-difference equation.

Let $\nabla_{q} f(z)=f(q z)-f(z)$,

then $\nabla_{q}\left(\nabla_{q}(f(z))=\mathrm{f}\left(\mathrm{q}^{2} z\right)-2 f(q z)+f(z)\right.$,

and hence $\nabla_{q}^{n} f(z)=\sum_{k=0}^{n} \frac{(-1)^{k} \cdot n !}{k ! \cdot(n-k) !} \cdot f\left(q^{k} z\right)$,

We define q-shift Monomial as

$$
M_{q}[f]=a(z) \prod_{j=0}^{k}\left[f^{(j)}(q z)\right]^{n} j
$$

where a(z) is small function, and $n_{j}$ are natural numbers, Then the degree of $\mathrm{M}_{q}[f]$ will be the sum of all the powers in the product on the right hand side. The weight of $\mathrm{M}_{q}[f]$ is defined as

$\Gamma_{M}=n_{0}+2 n_{1}+\ldots+(k+1) n_{k}$.

\section{DEFINITION 2 :}

Let

$$
M_{1}[f], M_{2}[f], \ldots
$$

denote the distinct q-shift monomials in $\mathrm{f}$, and

$$
a_{1}(z), a_{2}(z), \ldots
$$

be the small meromorphic functions including complex numbers then 


$$
P_{q}[f]=P_{q}[z, f]=\sum_{j \in \Delta} a_{j}(z) \cdot M_{j}[f]
$$

where $\Delta$ is a finite set of multi- indices, $a_{j}(z)$ are small functions of $\mathrm{f}, M_{j}[f]$ are q-shift monomials, will be called a q-shift differential polynomial in $\mathrm{f}$.

We define the total degree $\mathrm{d}$ and weight $\Gamma$ of $\mathrm{P}_{q}[z, f]$ in $\mathrm{f}$ as

$$
d=\underbrace{\operatorname{Max}}_{j \in \Delta} . d_{M_{j}}
$$

and $\Gamma=\underbrace{\operatorname{Max}}_{j \in \Delta} \Gamma_{M_{j}}$. If all the terms in the summation of $\mathrm{P}_{q}[f]$ have same degrees, then $\mathrm{P}_{q}[f]$ is known as homogeneous q-shift differential polynomial. Usually, we take $\mathrm{P}_{q}[f]$ such that $\mathrm{T}\left(\mathrm{r}, \mathrm{P}_{q}\right) \neq S(r, f)$.

Linear q-shift differential polynomial is of degree one e.g. $\nabla_{q}^{n} f(z)$.

\section{DEFINITION 3 :}

Let $\mathrm{k}$ be a positive integer and a be a complex number. We denote by $\left.N_{k}\right)(r, 1 /(f-$ $a)$ ), the counting function of a- points of $\mathrm{f}$ with multiplicity $\leq k$, by $N_{(k}(r, 1 /(f-$ $a)$ ), the counting function of a- points of $\mathrm{f}$ with multiplicity $\geq k$ then

Set $N_{k}(r, 1 /(f-a))=\bar{N}(r, 1 /(f-a))+\bar{N}_{(2}(r, 1 /(f-a))+\ldots+\bar{N}_{(k}(r, 1 /(f-a))$

Uniqueness Theory of Meromorphic functions is an important part of Nevanlinna Theory. Recently number of papers have focussed on the Nevanlinna Theory with respect to difference operators. This has lead to development of difference counterparts of many central results of Nevanlinna theory as more efficient tools to study difference equations. The development of this difference Nevanlinna theory has enabled us to further understand the value distribution of meromorphic functions without any connections to difference equations.

The classical result due to Nevanlinna theory of meromorphic functions is the five point theorem i.e. if two non-constant meromorphic functions $f$ and $g$ share five distinct values ignoring multiplicities(IM) then

$$
f(z) \equiv g(z)
$$

The number 5 is best possible. If the number of shared values is decreased, then an additional assumptions on value distribution needs to be introduced in order to obtain uniqueness.

A finite value a is called the Picard exceptional value of $\mathrm{f}$, if $\mathrm{f}$ - a has no zeros. The Picard theorem shows that a transcendental entire function has at most one 
Picard exceptional value, a transcendental meromorphic functions has at most two picard exceptional values. The classical problem of value distributions of differential polynomials is Hayman conjecture [1],i.e. if $\mathrm{f}$ is a transcendental meromorphic function and $\mathrm{n} \in \mathrm{N}$, then $\mathrm{f}^{n} f^{\prime}$ takes every finite non-zero value infinitely often which means that the Picard exceptional value of $\mathrm{f}^{n} f^{\prime}$ may only be zero. This conjecture has been proved by many authors. e.g., Hayman [3] proved that if $\mathrm{f}$ is a transcendental meromorphic function and $\mathrm{n} \geq 3$, then $\mathrm{f}^{n} f^{\prime}$ takes every finite non-zero complex value infinitely often. The case $n=2$ was proved by Mues[4], and Bergweiler et. al[5] proved the case for $n=1$.

Laine and Yang[6] has proved this conjecture for shifts and difference operators as following:

THEOREM A[6]: Let $\mathrm{f}$ be a transcendental entire function with finite order and $\mathrm{c}$ be a non-zero complex constant. Then for $\mathrm{n} \geq 2, \mathrm{f}(\mathrm{z})^{n} f(z+c)$ assumes every non-zero complex value a infinitely often.

Zhang, Korhonen[7] proved the analogous results in q-differences as follows:

THEOREM B[7]: Let $\mathrm{f}$ be a transcendental meromorphic function (resp. entire) with zero order and $q$ be a non-zero complex constant. Then for $n \geq$ 6 ( resp.n $\geq 2), \mathrm{f}(\mathrm{z})^{n} f(q z)$ assumes every non-zero complex value infinitely often.

THEOREM C[7]: Let $\mathrm{f}$ and $\mathrm{g}$ be two transcendental meromorphic functions (resp. entire) with zero order and $q$ be a non-zero complex constant.Then for $\mathrm{n}$ $\geq 8(\operatorname{resp} . \mathrm{n} \geq 4)$,if $\mathrm{f}(\mathrm{z})^{n} f(q z)$ and $\mathrm{g}(\mathrm{z})^{n} g(q z)$ share $1, \infty \mathrm{CM}$, then $\mathrm{f}(\mathrm{z})=\operatorname{tg}(\mathrm{z})$ for $t^{n+1}=1$.

Then many authors started to investigate the uniqueness of meromorphic functions sharing values with their shifts/ q-shifts or difference operators see e.g. $([8],[9])$.

Bergweiler et. al. [10] treated the functional equation $\sum_{j=0}^{n} a_{j}(z) f\left(c^{j} z\right)=Q(z)$ where $\mathrm{a}_{j}(z)$ and $Q(z)$ are rational functions, $a_{0} \neq 0$, $\mathrm{a}_{1}(z) \equiv 1,0<|c|<1$. They concluded that all meromorphic solutions of this equation are of zero order of growth.

We will consider the functional equation of the type

$\mathrm{f}^{l} \sum_{j=0}^{n} a_{j}(z) f\left(q^{j} z\right)=a(z)$ where $\mathrm{l} \in N, \mathrm{a}_{j}, j=0,1, \ldots, n$ and $\mathrm{a}(\mathrm{z}) \in S(f), a(z) \neq$ $0, \infty$ and prove the following results which are also generalizations of Theorem $\mathrm{B}$ and Theorem C:

\section{MAIN RESULTS:}

THEOREM 1.1: Let $\mathrm{f}$ be a transcendental meromorphic function with zero order and $\mathrm{P}_{q}[f]$ be a linear q-shift difference polynomial defined as

$P_{q}[f]=\sum_{j=0}^{n} a_{j}(z) f\left(q^{j} z\right), T\left(r, P_{q}[f]\right) \neq \mathrm{S}(\mathrm{r}, \mathrm{f}), q \neq 0$ then $f^{l} P_{q}[f]-a(z), a(z) \neq$ 
$0, \infty$ has infinitely many zeros provided $\mathrm{l}>4 \mathrm{n}+3$.

THEOREM 1.2: Let $\mathrm{f}$ be a transcendental entire function with zero order and $\mathrm{P}_{q}[f]$ be a linear q-shift difference polynomial defined as

$P_{q}[f]=\sum_{j=0}^{n} a_{j}(z) f\left(q^{j} z\right), T\left(r, P_{q}[f]\right) \neq \mathrm{S}(\mathrm{r}, \mathrm{f}), q \neq 0$ then $f^{l} P_{q}[f]-a(z), a(z) \neq$ $0, \infty$ has infinitely many zeros provided $\mathrm{l}>2 \mathrm{n}+1$.

THEOREM 1.3: Let $\mathrm{f}$ be a transcendental meromorphic function with zero order and

$$
P_{q}[f]=P_{q}[z, f]=\sum_{j \in \Delta} a_{j}(z) \cdot M_{j}[f]
$$

where $\Delta$ is a finite set of multi- indices, $a_{j}(z)$ are small functions of $\mathrm{f}, M_{j}[f]$ are q-shift monomials, be a q-shift differential polynomial in $\mathrm{f}, \mathrm{T}\left(\mathrm{r}, \mathrm{P}_{q}[f]\right) \neq \mathrm{S}(\mathrm{r}, \mathrm{f})$, $q \neq 0$ then $f^{l} P_{q}[f]-a(z), a(z) \neq 0, \infty$ has infinitely many zeros provided $l>$ $2(\Gamma+1)$.

\section{COROLLARIES:}

1. If $\mathrm{n}=0$ in above theorems 1.1 and $1.2, \mathrm{P}_{q}[f]=f(q z)$, and $\mathrm{a}(\mathrm{z})$ as nonzero complex values, then we get improvement of Theorem $\mathrm{B}$ as following: i. for transcendental entire function $\mathrm{f}, \mathrm{f}^{l} f(q z)$ assumes every non-zero complex value 'a' infinitely often provided $l>1$.

ii. In case of transcendental meromorphic function $\mathrm{f}, \mathrm{f}^{l} f(q z)$ assumes every non-zero and finite complex value 'a' infinitely often provided $l>3$, whereas in Theorem $\mathrm{B}, \mathrm{l} \geq 6$.

2. If $\mathrm{n}=1$ in above theorems 1.1 and 1.2 , we take $\mathrm{P}_{q}[f]=f(q z)-f(z)$, and $\mathrm{a}(\mathrm{z})$ as non-zero complex values, then

i. for transcendental entire function $\mathrm{f}, \mathrm{f}^{l}[f(q z)-f(z)](\neq S(r, f))$ assumes every non-zero complex value 'a' infinitely often provided $l>3$.

ii. In case of transcendental meromorphic function $\mathrm{f}, \mathrm{f}^{l}[f(q z)-f(z)](\neq S(r, f))$ assumes every non-zero and finite complex value 'a' infinitely often provided 1 $>7$, whereas by Theorem 1.3, the results holds for $l>4$ which is better.

3.The results can be obtained for $\mathrm{f}^{l} \nabla_{q}^{n} f(z)=\mathrm{f}^{l} \sum_{k=0}^{n} \frac{(-1)^{k} \cdot n !}{k ! \cdot(n-k) !} \cdot f\left(q^{k} z\right)$, when we put $a_{j}(z)=\frac{(-1)^{j} \cdot n !}{j ! !(n-j) !}$ in $\mathrm{P}_{q}[f]$.

For the proof of the results we need the following lemmas:

LEMMA 1 [7]: Let $\mathrm{f}$ be a non- constant meromorphic function of zero order and $q$ be a non- zero complex constant, then

$$
m\left(r, \frac{f(q z)}{f(z)}\right)=\mathrm{S}(\mathrm{r}, \mathrm{f}),
$$


for all $\mathrm{r}$ outside a possible exceptional set of finite logarithmic measure.

LEMMA 2[7]: Let q be the non-zero complex constant, and let $\mathrm{f}$ be a meromorphic function of zero order then

$$
\begin{aligned}
& \mathrm{T}(\mathrm{r}, \mathrm{f}(\mathrm{qz}))=\mathrm{T}(\mathrm{r}, \mathrm{f})+\mathrm{S}(\mathrm{r}, \mathrm{f}) \\
& \mathrm{N}(\mathrm{r}, \mathrm{f}(\mathrm{qz}))=\mathrm{N}(\mathrm{r}, \mathrm{f})+\mathrm{S}(\mathrm{r}, \mathrm{f}) \\
& \mathrm{N}(\mathrm{r}, 0, \mathrm{f}(\mathrm{qz}))=\mathrm{N}(\mathrm{r}, 0, \mathrm{f})+\mathrm{S}(\mathrm{r}, \mathrm{f})
\end{aligned}
$$

LEMMA 3([11]): LetF and G be two non-constant meromorphic functions. If $\mathrm{F}$ and $\mathrm{G}$ share $1 \mathrm{CM}$, then one of the following three cases holds:

i. $\max$. ( T(r, F), T(r, G) $) \leq N_{2}(r, 0, F)+N_{2}(r, 0, G)+N_{2}(r, F)+N_{2}(r, G)+$ $S(r, F)+S(r, G)$

$i i . F \equiv G$

iii.F.G $\equiv 1$.

LEMMA 4([12]): Let $\mathrm{f}$ be a nonconstant meromorphic function. If $\mathrm{Q}[\mathrm{f}]$ is a differential polynomial in $\mathrm{f}$ with arbitrary meromorphic coefficients, then

( i) $\mathrm{m}(\mathrm{r}, \mathrm{Q}[\mathrm{f}]) \leq \gamma_{Q} m(r, f)+\mathrm{S}(\mathrm{r}, \mathrm{f})$

(ii) $\left.\mathrm{N}(\mathrm{r}, \mathrm{Q}[\mathrm{f}]) \leq \Gamma_{Q} N r, f\right)+S(r, f)$

\section{PROOF OF THEOREM 1.1:}

Let $\mathrm{G}[\mathrm{z}]=\mathrm{f}^{l} P_{q}[f]$ where $\mathrm{f}$ is a meromorphic function and suppose $\mathrm{G}[\mathrm{z}]-\mathrm{a}(\mathrm{z})$, $\mathrm{a}(\mathrm{z}) \neq 0, \infty$ has finitely many zeros. Then we get by using Lemma 1 and Lemma 2

$$
\begin{aligned}
& \mathrm{T}(\mathrm{r}, \mathrm{G}[\mathrm{z}])=\mathrm{T}\left(\mathrm{r}, \mathrm{f}^{l}\left[a_{0} f(z)+a_{1} f(q z)+a_{2} f\left(q^{2} z\right)+\ldots+a_{n} f\left(q^{n} z\right)\right]\right) \\
& =\mathrm{T}\left(\mathrm{r}, \mathrm{f}^{l+1}\left[a_{0}+a_{1} \frac{f(q z)}{f(z)}+a_{2} \frac{f\left(q^{2} z\right)}{f(z)}+\ldots+a_{n} \frac{f\left(q^{n} z\right)}{f(z)}\right]\right. \\
& \geq(\mathrm{l}+1) \mathrm{T}(\mathrm{r}, \mathrm{f})-\mathrm{T}\left(\mathrm{r},\left[\mathrm{a}_{0}+a_{1} \frac{f(q z)}{f(z)}+a_{2} \frac{f\left(q^{2} z\right)}{f(z)}+\ldots+a_{n} \frac{f\left(q^{n} z\right)}{f(z)}\right]\right) \\
& \text { But } \mathrm{T}\left(\mathrm{r},\left[\mathrm{a}_{0}+a_{1} \frac{f(q z)}{f(z)}+a_{2} \frac{f\left(q^{2} z\right)}{f(z)}+\ldots+a_{n} \frac{f\left(q^{n} z\right)}{f(z)}\right]\right) \\
& \leq \mathrm{T}\left(\mathrm{r}, \frac{f(q z)}{f(z)}\right)+\mathrm{T}\left(\mathrm{r}, \frac{f\left(q^{2} z\right)}{f(z)}\right)+\ldots+\mathrm{T}\left(\mathrm{r}, \frac{f\left(q^{n} z\right)}{f(z)}\right)+\mathrm{S}(\mathrm{r}, \mathrm{f}) \\
& =\mathrm{N}\left(\mathrm{r}, \frac{f(q z)}{f(z)}\right)+\mathrm{N}\left(\mathrm{r}, \frac{f\left(q^{2} z\right)}{f(z)}\right)+\ldots+\mathrm{N}\left(\mathrm{r}, \frac{f\left(q^{n} z\right)}{f(z)}\right)+\mathrm{S}(\mathrm{r}, \mathrm{f})
\end{aligned}
$$


$\leq 2 \mathrm{n} \mathrm{T}(\mathrm{r}, \mathrm{f})+\mathrm{S}(\mathrm{r}, \mathrm{f})$

Therefore, we have

$\mathrm{T}(\mathrm{r}, \mathrm{G}[\mathrm{z}])$
$\geq(\mathrm{l}+1) \mathrm{T}(\mathrm{r}, \mathrm{f})-2 \mathrm{nT}(\mathrm{r}, \mathrm{f})+\mathrm{S}(\mathrm{r}, \mathrm{f})$

Since $\mathrm{f}$ is meromorphic, therefore, by using Nevanlinna's second main theorem and lemma, we get

$$
\begin{aligned}
& {[\mathrm{l}+1-2 \mathrm{n}] \mathrm{T}(\mathrm{r}, \mathrm{f}) \leq \mathrm{T}(\mathrm{r}, \mathrm{G}[\mathrm{z}]) \leq \bar{N}\left(r, \frac{1}{G(z)}\right)+\bar{N}(r, G(z))+\bar{N}\left(r, \frac{1}{G(z)-a(z)}\right) } \\
+ & \mathrm{S}(\mathrm{r}, \mathrm{G}) \\
& =\bar{N}\left(r, \frac{1}{G(z)}\right)+\bar{N}(r, G(z))+S(r, f) \\
& \leq(2 \mathrm{n}+4) \mathrm{T}(\mathrm{r}, \mathrm{f})+S(r, f)
\end{aligned}
$$

So we get

$$
\mathrm{l} \mathrm{T}(\mathrm{r}, \mathrm{f}) \leq(4 \mathrm{n}+3) \mathrm{T}(\mathrm{r}, \mathrm{f})+S(r, f)
$$

which is a contradiction as $1>4 \mathrm{n}+3$. Thus our supposition is wrong and hence, $f^{l} P_{q}[f]-a(z), a(z) \neq 0, \infty$ has infinitely many zeros.

\section{PROOF OF THEOREM 1.2:}

Let $\mathrm{G}[\mathrm{z}]=\mathrm{f}^{l} P_{q}[f]$ where $\mathrm{f}$ is an entire function and suppose $\mathrm{G}[\mathrm{z}]-\mathrm{a}(\mathrm{z}), \mathrm{a}(\mathrm{z}) \neq 0$, $\infty$ has finitely many zeros. Then we get by using Lemma 1 and Lemma 2

$$
\begin{aligned}
& \mathrm{T}(\mathrm{r}, \mathrm{G}[\mathrm{z}])=\mathrm{T}\left(\mathrm{r}, \mathrm{f}^{l}\left[a_{0} f(z)+a_{1} f(q z)+a_{2} f\left(q^{2} z\right)+\ldots+a_{n} f\left(q^{n} z\right)\right]\right) \\
& =\mathrm{T}\left(\mathrm{r}, \mathrm{f}^{l+1}\left[a_{0}+a_{1} \frac{f(q z)}{f(z)}+a_{2} \frac{f\left(q^{2} z\right)}{f(z)}+\ldots+a_{n} \frac{f\left(q^{n} z\right)}{f(z)}\right]\right. \\
& \geq(\mathrm{l}+1) \mathrm{T}(\mathrm{r}, \mathrm{f})-\mathrm{T}\left(\mathrm{r},\left[\mathrm{a}_{0}+a_{1} \frac{f(q z)}{f(z)}+a_{2} \frac{f\left(q^{2} z\right)}{f(z)}+\ldots+a_{n} \frac{f\left(q^{n} z\right)}{f(z)}\right]\right) \\
& \text { But } \mathrm{T}\left(\mathrm{r},\left[\mathrm{a}_{0}+a_{1} \frac{f(q z)}{f(z)}+a_{2} \frac{f\left(q^{2} z\right)}{f(z)}+\ldots+a_{n} \frac{f\left(q^{n} z\right)}{f(z)}\right]\right) \\
& \leq \mathrm{T}\left(\mathrm{r}, \frac{f(q z)}{f(z)}\right)+\mathrm{T}\left(\mathrm{r}, \frac{f\left(q^{2} z\right)}{f(z)}\right)+\ldots+\mathrm{T}\left(\mathrm{r}, \frac{f\left(q^{n} z\right)}{f(z)}\right)+\mathrm{S}(\mathrm{r}, \mathrm{f}) \\
& =\mathrm{N}\left(\mathrm{r}, \frac{f(q z)}{f(z)}\right)+\mathrm{N}\left(\mathrm{r}, \frac{f\left(q^{2} z\right)}{f(z)}\right)+\ldots+\mathrm{N}\left(\mathrm{r}, \frac{f\left(q^{n} z\right)}{f(z)}\right)+\mathrm{S}(\mathrm{r}, \mathrm{f}) \\
& =\mathrm{N}\left(\mathrm{r}, \frac{1}{f(z)}\right)+\mathrm{N}\left(\mathrm{r}, \frac{1}{f(z)}\right)+\ldots+\mathrm{N}\left(\mathrm{r}, \frac{1}{f(z)}\right)+\mathrm{S}(\mathrm{r}, \mathrm{f}) \\
& \leq \mathrm{n} \mathrm{T}(\mathrm{r}, \mathrm{f})+\mathrm{S}(\mathrm{r}, \mathrm{f})
\end{aligned}
$$


Therefore, we have

$$
\begin{aligned}
& \mathrm{T}(\mathrm{r}, \mathrm{G}[\mathrm{z}]) \\
& \geq(\mathrm{l}+1) \mathrm{T}(\mathrm{r}, \mathrm{f})-\mathrm{n} \mathrm{T}(\mathrm{r}, \mathrm{f})+\mathrm{S}(\mathrm{r}, \mathrm{f})
\end{aligned}
$$

Since $\mathrm{f}$ is entire, therefore, by using Nevanlinna's second main theorem and lemma, we get

$$
\begin{aligned}
& {[\mathrm{l}+1-\mathrm{n}] \mathrm{T}(\mathrm{r}, \mathrm{f}) \leq \mathrm{T}(\mathrm{r}, \mathrm{G}[\mathrm{z}]) \leq \bar{N}\left(r, \frac{1}{G(z)}\right)+\bar{N}\left(r, \frac{1}{G(z)-a(z)}\right)+\mathrm{S}(\mathrm{r}, \mathrm{G})} \\
& =\bar{N}\left(r, \frac{1}{G(z)}\right)+\mathrm{S}(\mathrm{r}, \mathrm{f}) \\
& \leq(\mathrm{n}+2) \mathrm{N}(\mathrm{r}, 0, \mathrm{f})+S(r, f) \\
& \leq(\mathrm{n}+2) \mathrm{T}(\mathrm{r}, \mathrm{f})+S(r, f)
\end{aligned}
$$

So we get

$$
\mathrm{l} \mathrm{T}(\mathrm{r}, \mathrm{f}) \leq(2 \mathrm{n}+1) \mathrm{T}(\mathrm{r}, \mathrm{f})+S(r, f)
$$

which is a contradiction as $1>2 \mathrm{n}+1$. Thus our supposition is wrong and hence, $\mathrm{f}^{l} P_{q}[f]-a(z), a(z) \neq 0, \infty$ has infinitely many zeros.

\section{PROOF OF THEOREM 1.3:}

Let $\mathrm{G}[\mathrm{z}]=\mathrm{f}^{l} P_{q}[z, f]$ where $\mathrm{f}$ is a meromorphic function and suppose $\mathrm{G}[\mathrm{z}]-\mathrm{a}(\mathrm{z})$, $\mathrm{a}(\mathrm{z}) \neq 0, \infty$ has finitely many zeros. Then we get by using Lemma 1 and Lemma 4

$$
\begin{aligned}
& \mathrm{T}(\mathrm{r}, \mathrm{G}[\mathrm{z}])=\mathrm{T}\left(\mathrm{r}, \mathrm{f}^{l} \sum_{j \in \Delta} a_{j}(z) \cdot M_{j}[f]\right) \\
& \geq \mathrm{lT}(\mathrm{r}, \mathrm{f})-\Gamma_{Q} T(r, f)
\end{aligned}
$$

Therefore, we have

$$
\mathrm{T}(\mathrm{r}, \mathrm{G}[\mathrm{z}])
$$

$\geq\left(1-\Gamma_{Q}\right) T(r, f)+S(r, f)$

Since $\mathrm{f}$ is meromorphic, therefore, by using Nevanlinna's second main theorem and lemma, we get $\mathrm{S}(\mathrm{r}, \mathrm{G})$

$\left(\mathrm{l}-\Gamma_{Q}\right) T(r, f) \leq \mathrm{T}(\mathrm{r}, \mathrm{G}[\mathrm{z}]) \leq \bar{N}\left(r, \frac{1}{G(z)}\right)+\bar{N}(r, G(z))+\bar{N}\left(r, \frac{1}{G(z)-a(z)}\right)+$ 


$$
\begin{aligned}
& =\bar{N}\left(r, \frac{1}{G(z)}\right)+\bar{N}(r, G(z))+S(r, f) \\
& \leq\left(\Gamma_{Q}+1\right) T(r, f)+\bar{N}(r, f)+S(r, f) \\
& =\left(\Gamma_{Q}+2\right) T(r, f)+S(r, f)
\end{aligned}
$$

So we get

$\mathrm{l} \mathrm{T}(\mathrm{r}, \mathrm{f}) \leq 2\left(\Gamma_{Q}+1\right) T(r, f)+\mathrm{S}(\mathrm{r}, \mathrm{f})$

which is a contradiction as $1>2\left(\Gamma_{Q}+1\right)$. Thus our supposition is wrong and hence the result.

\section{APPLICATIONS:}

As applications of the above main results, we present the following result:

THEOREM 2.1: Let $f$ and $g$ be transcendental entire functions with finite order and as in definition $2, \mathrm{P}_{q}[f]$ and $\mathrm{P}_{q}[g]$ be two linear q-shift difference polynomials and $f^{l} P_{q}[f]$ and $g^{l} P_{q}[g]$ share $\mathrm{a}(\mathrm{z}), \mathrm{a}(\mathrm{z}) \neq 0, \infty \mathrm{CM}$, then $f^{l} P_{q}[f]=g^{l} P_{q}[g]$ or $f^{l} P_{q}[f] . g^{l} P_{q}[g]=(a(z))^{2}$. provided $\mathrm{l}>3 \mathrm{n}+5$.

\section{PROOF OF THEOREM 2.1:}

Let $\mathrm{F}(\mathrm{z})=\frac{f^{l} P_{q}[f]}{a(z)}$ and $\mathrm{G}(\mathrm{z})=\frac{g^{l} P_{q}[g]}{a(z)}$, then $\mathrm{F}(\mathrm{z})$ and $\mathrm{G}(\mathrm{z})$ share $1 \mathrm{CM}$ except the zeros or poles of $\mathrm{a}(\mathrm{z})$. We have by using Lemma 3 ,

$$
\begin{aligned}
& \mathrm{N}_{2}(r, 0, F)=N_{2}\left(r, 0, f^{l}\right)+N_{2}\left(r, 0, P_{q}[f]\right)+S(r, f) \\
& \leq N_{1)}(r, 0, f)+N(r, 0, f)+N\left(r, 0, P_{q}[f]\right)+S(r, f) \text { by definition } 3 \\
& \leq(n+3) T(r, f)+S(r, f)
\end{aligned}
$$

Similarly, we have $\mathrm{N}_{2}(r, 0, G) \leq(n+3) T(r, g)+S(r, g)$

By Lemma 3, suppose i, holds, then since f, g are entire functions

$$
\begin{aligned}
& \max .(\mathrm{T}(\mathrm{r}, \mathrm{F}), \mathrm{T}(\mathrm{r}, \mathrm{G})) \leq N_{2}(r, 0, F)+N_{2}(r, 0, G)+N_{2}(r, F)+N_{2}(r, G)+ \\
& S(r, F)+S(r, G) \\
& \quad \leq(n+3)[T(r, f)+T(r, g)]+S(r, f)+S(r, g)
\end{aligned}
$$

Thus, we have

$$
\mathrm{T}(\mathrm{r}, \mathrm{F})+\mathrm{T}(\mathrm{r}, \mathrm{G}) \leq 2(n+3)[T(r, f)+T(r, g)]+S(r, f)+S(r, g)
$$


Using eq. 1 we have

$[\mathrm{l}+1-\mathrm{n}][\mathrm{T}(\mathrm{r}, \mathrm{f})+\mathrm{T}(\mathrm{r}, \mathrm{g})] \leq \mathrm{T}(\mathrm{r}, \mathrm{F})+\mathrm{T}(\mathrm{r}, \mathrm{G})$

$\leq 2(n+3)[T(r, f)+T(r, g)]+S(r, f)+S(r, g)$

Thus, $l .[T(r, f)+T(r, g)] \leq(3 n+5)[T(r, f)+T(r, g)]+S(r, f)+S(r, g)$

which contradicts the given condition that $1>3 n+5$.

Hence by Lemma 3, result holds.

REMARK: Similar result can be proved when $\mathrm{f}$, $\mathrm{g}$ are meromorphic functions.

\section{COROLLARIES:}

1. Let $f$ and $g$ be transcendental entire functions with finite order, $q$ be non-zero complex constant and if $\mathrm{F}=\mathrm{f}^{n} f(q z)$ and $\mathrm{G}=\mathrm{g}^{n} g(q z)$ share $1 \mathrm{CM}$, then for $n>5, \mathrm{~F} \equiv G$ or $\mathrm{F} . \mathrm{G} \equiv 1$.

2. Let $\mathrm{f}$ and $\mathrm{g}$ be transcendental entire functions with finite order, $\mathrm{q}$ be non-zero complex constant and if $\mathrm{F}=\mathrm{f}^{n}[f(q z)-f(z)]$ and $\mathrm{G}=\mathrm{g}^{n}[g(q z)-g(z)]$ share 1 $\mathrm{CM}$, then for $n>8, \mathrm{~F} \equiv G$ or $\mathrm{F} . \mathrm{G} \equiv 1$.

3. Similar results can be obtained for $\mathrm{f}^{l} \nabla_{q}^{n} f(z)$ and $\mathrm{g}^{l} \nabla_{q}^{n} g(z)$ for all $\mathrm{n}$.

\section{EXAMPLE:}

The zero order in main results cannot be extended to finite order as shown in following example:

$\mathrm{f}(\mathrm{z})=\mathrm{e}^{z}, q=-n$ then $\mathrm{f}^{n} \cdot f(q z) \equiv 1$.

\section{Conflict of interest:}

The author has no conflict of interest to declare.

\section{REFERENCES:}

1. Hayman, W.K.: Meromorphic functions- Clarenden Press, Oxford, 1964.

2. Yi, H.X., Yang, C.C.: Uniqueness Theory of Meromorphic Functions- Kluwer Academic, Dordrecht (2003).[Chinese original: Science Press, Beijing (1995)].

3. Hayman, W.K.: Picard values of meromorphic functions and their derivativesAnn. of Math. (2), 70 (1959), pp.9-42.

4. Mues E, ber ein: Problem von Hayman- Math. Z. 164 (1979), pp.239259 . 
5. Bergweiler, W. and Eremenko, A.: On the singularities of the inverse to a meromorphic function of finite order- Revista Matemtica Iberoamericana, 11 (1995), pp.355-373.

6. Laine, I., Yang, C.C.: Value distribution and uniqueness of difference polynomials -Proc. Japan Acad. Ser. A, 83(2007), pp.148-151.

7. Zhang, J. and Korhonen,R.: On the Nevanlinna characteristic of $\mathrm{f}(\mathrm{qz})$ and its applications- Journal of Mathematical Anal. and Appl., vol. 369, no. 2, 2010, pp.537-544.

8. Barnett,D., Halburd,R.J., Korhonen, Morgan: Applications of Nevanlinna theory to q-difference equations- Proc. Roy. Soc. Edinburgh Sect. A 137(2007), pp.457-474.

9. Liu, K., Liu, X., Cao, T.B. : Value distribution of the difference operator, Advances in Difference equations, Vol.2011, Art ID 234215,12 pages.

10. W. Bergweiler, K. Ishizaki, N. Yanagihara, Meromorphic solutions of some functional equations, Methods Appl. Anal. 5 (1998), pp.248-258, correction:Methods Appl. Anal. 6 (1999).

11. Yang, C.C., Hua, X.H.: Uniqueness and value sharing of meromorphic functions, Ann. Acad.Sci. Fenn. Math. 22(1997), pp.395-406.

12. Doeringer, W.: Exceptional values of Differential Polynomials, Pacific J. of Math., 98(no. 1) 1982, pp.55-62. 